\title{
VOTING-BASED ACTIVE CONTOUR SEGMENTATION OF FMRI IMAGES OF THE BRAIN
}

\author{
Gowri Srinivasa ${ }^{1}$, Vivek S. Oak ${ }^{2}$, Siddharth J. Garg ${ }^{2}$, Matthew C. Fickus ${ }^{3}$ and Jelena Kovačević ${ }^{1,2}$ \\ ${ }^{1}$ Dept. of Biomedical Eng., ${ }^{2}$ Dept. of Electrical and Computer Eng., \\ Carnegie Mellon University, Pittsburgh, PA, USA \\ ${ }^{3}$ Dept. of Math. and Statistics, Air Force Inst. of Tech., Wright-Patterson AFB, OH, USA
}

\begin{abstract}
We propose an algorithm for automated segmentation of white matter in brain MRI images, which can be used to create connected representations of the gray matter in the cerebral cortex of the brain. These representations then provide meaningful visualizations of brain activity data obtained from fMRI studies. Our algorithm to segment the white matter from the rest of the image is based on an active-contour scheme-STACS, and thus inherits all the advantages active-contour schemes possess. The segmentation, performed in three different planes of image capture, is driven by the statistics of the image. We combine the segmentation results from the three planes by a majority voting procedure to classify each voxel in the image as white matter or not. We improve the runtime of the algorithm by rewriting the force computation as a multiscale transformation. Initial results of labeling the white matter with an accuracy of about $89 \%$ show great promise of the proposed algorithm.
\end{abstract}

Index Terms - brain fMRI, segmentation, active contour.

\section{INTRODUCTION}

Magnetic Resonance Imaging (MRI) has emerged as a powerful technique to noninvasively visualize biological structures. Furthermore, with the development of functional MRI (fMRI) techniques, it is possible to directly visualize neuronal activity in the brain by using the amount of oxygen in the surrounding blood flow as a proxy for neuronal activity.

Anatomical MRI images of the whole human brain are collected routinely at a resolution of about $1 \mathrm{~mm}^{3}$ in functional and anatomical imaging studies. Segmentation of these MRI brain images into gray and white matter, or, tissue types, is crucial for multiple purposes, including: (a) measuring the location and size of visual field maps in human occipital cortex by overlaying fMRI signals on surface representations of the gray matter [1]; (b) studying the organization of retinotopic, motion and object sensitive cortex through 3D visualization of an inflated surface along the gray-white boundary of the brain [2], (c) mapping gray matter growth and loss patterns in normal development and in neurological conditions by computing deformation fields on the entire gray matter mask or on specific structures (for example, lateral ventricles, corpus callosum) defined by tissue type (respectively: CSF, white matter) [3], and many others. Thus, high quality tissue segmentation is a powerful tool that enables multiple lines of investigation in neuroscience [4].

While it is possible for human experts to label each voxel in a 3D MRI image of the brain by hand, the time and effort can be

This work was supported in part by NSF through awards ITR-EF0331657 and DMS-0405376 as well as the PA State Tobacco Settlement, Kamlet-Smith Bioinformatics Grant. prohibitive. Furthermore, automated techniques guarantee reproducible results. Finally, state-of-the-art generic automated segmentation techniques that do not take into account the specific nature of brain MRI images and the topological features of the cerebral cortex can not yield sufficient accuracy to match manual segmentation results. This motivates the need to consider algorithms that can incorporate domain knowledge of the task at hand (prior knowledge of the topology of the brain in this case).

Current segmentation algorithms used on 3D brain MRI images to extract the three classes of cerebral tissue - the gray matter, white matter and CSF - can be classified into two major categories: Voxel-based classification and deformable or active-contour-based segmentation. Voxel-based classification methods use image statistics and histogram-based thresholding $[5,6]$. While partially successful, these methods are affected by intensity variations, the need to maintain distinct templates for various brain pathologies when coregistration with a template is incorporated, and finally, they do not necessarily incorporate the connectedness of the brain tissue. Active-contour-based methods, on the other hand, have considerable advantages due to their topological flexibility $[7,8]$.

In this paper, we propose an algorithm based on the stochastic active contour algorithm (STACS), which combines the advantages of active contour schemes with the incorporation of model statistics from the image $[9,10]$. The algorithm we propose harnesses the three-dimensional information that is available in the brain MRI image to improve the robustness and quality of the $2 \mathrm{D}$ segmentation produced by the suitably adapted STACS.

Organization of the paper. Section 2 gives the necessary background information on the structure of the brain in the context of the segmentation problem and the algorithm used to obtain an initial segmentation, a short description of a publicly available algorithm mrGray, as well as a short introduction into STACS. This is followed by Section 3 that describes our proposed algorithm-the voting-based STACS and an improvement in its runtime using a multiscale active contour (MSAC) transformation. In Section 5, we discuss our experimental results.

\section{BACKGROUND}

To generate meaningful visualizations of the data obtained from fMRI studies, one must first obtain accurate topological maps of the area of the brain in which the neuronal activity is being measured-the gray matter in the cerebral cortex. One way to create these topological maps is to segment 3D MRI images of the brain into three regions-gray matter, white matter and cerebral spinal fluid (CSF). Thus, it is necessary to understand the structure of the brain and in particular, the topology of the cerebral cortex (gray matter) and the surrounding white matter and cerebral spinal fluid in the context of segmentation. 
Structure of the Brain. Gray matter forms the outer layer of the brain (called the cortex), completely encasing the inner white matter. The gray matter is highly folded to allow a higher surface area to volume ratio, and its topology resembles "two crumpled sheets having no holes or self intersections" [11]. Further, a large portion of the gray matter resides in the deep fissures, or, sulci, that surround the ridges on the surface of the brain. On the other hand, white matter forms the bulk of the deep parts of the brain, and is a large monolithic structure with no holes (see Fig. 1). Finally, the cerebral spinal fluid (CSF) occupies the region between the cerebral cortex and the skull and appears in brain MRI images as a black region that surrounds the gray matter.

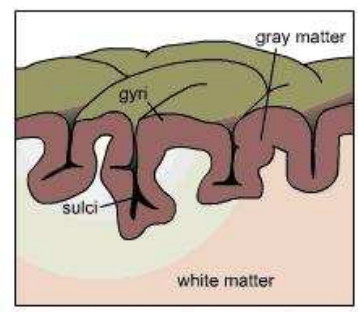

Fig. 1. Cross section of the brain showing the gray matter and white matter [12].

Based on the specific characteristics of brain MRI images and on the topology of the brain, there are two problems that complicate the brain image segmentation task [11]. The first is that there is a significant overlap between the intensities of the gray and white matter voxels, thereby making it impossible to segment images based on voxel intensity alone. Next, the presence of narrow sulci makes it hard to determine the connectivity of gray-matter regions, since gray-matter voxels on either side of a sulcus may appear to be connected if the sampling grid is sparse.

mrGray. A technique for brain MR image segmentation that uses prior knowledge of the structure of the human brain to aid the segmentation process was proposed in [11] and made publicly available as a MATLAB toolbox called mrGray [13]. Due to the difficulties in segmenting the gray matter directly, the authors chose to segment only the white matter and CSF regions in the image. Once the white matter has been segmented, the proposed technique reconstructs the gray matter surface using a method of "constrained growing-out from the white matter boundary". White matter segmentation is performed by building statistical models of pixel intensities for the white matter and nonwhite matter regions of the brain followed by a step that assigns, to each pixel intensity value, a label based on a novel maximum aposteriori probability (MAP) estimation algorithm. However, the authors observe that the segmented white matter regions obtained from this step occasionally have holes and handles within them. Since actual white matter regions cannot possess such holes or handles, all voxels that lie in these holes or handles are relabelled as white matter. Finally, layers of gray matter are grown iteratively, starting from the edge of the white matter region. The number of layers of gray matter that need to be grown is taken as an input from the user. Care is taken to ensure the connectivity of gray matter regions within and across layers. In the algorithm, the quality of the white matter segmentation directly affects the quality of the final 3D gray matter topology. In this paper, we only address the issue of the white matter segmentation using an active-contour based approach, as opposed to the MAP-based approach used in [11]. The improved segmentation can then be used as an input to the hole-filling and gray matter growing steps of mrGray.

Active contours. Active contours is a flexible and adaptive class of algorithms that evolved from the need to find a method that looks for any shape in the image that is smooth and forms a closed contour around it. The Stochastic Active Contour Scheme(STACS) was introduced in [9] to take into account the specific nature of the segmentation problem in cardiac MR images. STACS adjusts the shape of an initial curve (or contour) based on internal and external forces until it reaches equilibrium. The original STACS algorithm uses four forces: a region-based force, an edge-based force, a shape prior and the contour smoothness. These forces are suggestive as they are application specific and may be suitably included, if required. STACS uses a level set embedding in which the contour is the zero level of a level-set function $\phi(x, y, t)$.

\section{PROPOSED ACTIVE CONTOUR SEGMENTATION}

As a first step, we adapted STACS to segment the white matter in brain MR images. Subsequently, we improved the runtime of the algorithm by rewriting the level-set evolution (force computation) as a MSAC transformation.

\subsection{Voting-based STACS}

The steps of the proposed voting-based algorithm for the white matter segmentation of white matter are described below.

Initial Contours. We threshold the input image $f$, using an empirically determined threshold value and then apply a series of morphological operations to reduce the number of initial contours obtained and retain a meaningful result. This circumvents the need for manual intervention in initializing the contour (as required for STACS). The initial contours evolve based on the forces applied on them.

Choice of Forces. Since the model statistics of the MRI image are fairly strong, with the white matter being fairly distinct from the nonwhite matter, we chose a region-based force to drive the segmentation. To compute the actual force, we first represent both the white matter and the nonwhite matter by their respective statistical models. We compute the statistical models by selecting a few representative slices from the volume as training data and computing the discriminating statistics for the foreground and background regions. We exclude the images used as training data later in the testing phase to quantify the algorithm's performance. The two statistical models are based on the mean pixel intensities of the white matter (foreground) and nonwhite matter (background) regions. The foreground and background regions exhibit significant intensity variations, with the regions near the edges exhibiting lower or higher intensities than the means of their respective regions. Therefore, while deriving the model statistics from the training images, we compute the model mean intensities using only small windows around the foregroundbackground edges (specifically, the edge between the white and nonwhite matter). The evolution of the level-set function for a point $(x, y)$ on the contour at a time step $t$ can now be described as

$$
\frac{\partial \phi(x, y, t)}{\partial t}=\lambda_{r} F_{r}(x, y)|\nabla \phi(x, y, t)|
$$

where the region-based force $F_{r}(x, y)$ is computed only on points along the contour and subsequently extended to the whole domain. $\lambda_{r}$ is a weighting function that modulates the strength of the regionbased force as the contour evolves. This ensures that the contour changes shape rapidly in the beginning of its evolution and gradually slows down as it approaches its desired location. The value of $\lambda_{r}$ is determined in every iteration using the annealing schedule $S_{r}$ 
proposed in [9]. The shape and smoothness-based forces are inapplicable since the white matter region possesses neither smooth edges nor a well-defined shape that can be used as a template. Finally, while the edge-based force seemed like a good candidate for inclusion, it actually degraded the quality of our final segmentation due to the existence of a number of spurious edges (such as the edges between the gray matter and CSF regions) in the edge map of the image. Therefore we omitted the edge-based force from the algorithm.

Voting. The original STACS implementation and the discussion until now implicitly assumed segmentation on a 2D data set, while the brain MR image data set is actually three-dimensional. The additional information available along the third dimension can be harnessed to improve the robustness of the algorithm and the results of the 2D segmentation. A given 3D image can be sliced into a collection of parallel 2D images along three orthogonal axes or planes (commonly referred to as axial, coronal and sagittal planes by biologists). While performing segmentation on a collection of 2D slices from any one plane is sufficient to label each voxel as either white matter or nonwhite matter, we can build some redundancy into the system by segmenting all slices along each one of the three planes. Since there are three orthogonal planes that pass through each voxel in a 3D image, the segmentation result of each of these three planes would independently label a voxel as either white matter or nonwhite matter. The "vote" from each plane could then be combined using a simple majority voting procedure to yield the final label for that voxel. The pseudo code for the algorithm is presented in Algorithm 1 .

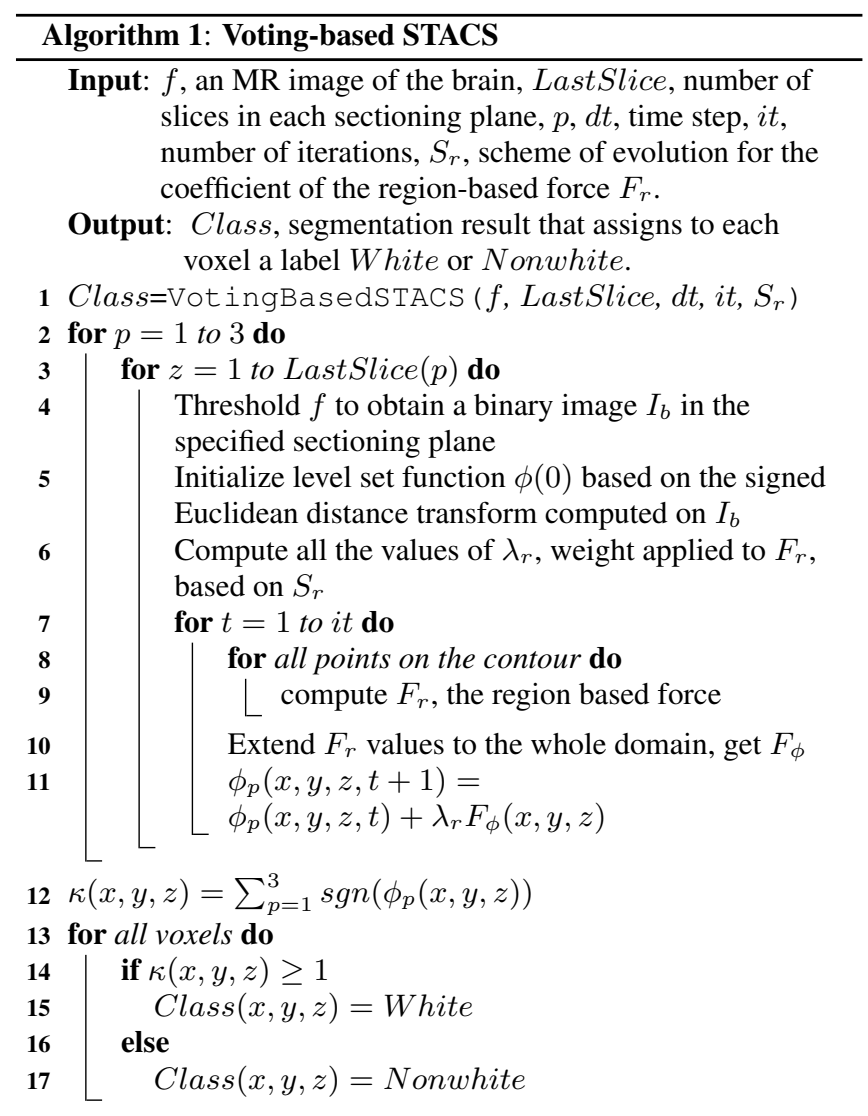

\subsection{MSAC Transformation}

A profile of the implementation revealed that the bottleneck in the implementation of Algorithm 1 was the step that updated the levelset function by extending the forces computed only at points on the contour to the entire domain. It has been shown that using the levelset function to merely track the foreground and background regions is more efficient than strictly using a signed distance interpretation of the function and evolving it in small steps $[14,15]$. Thus, inspired by the these results, we rewrote the algorithm so as to compute the forces everywhere and thus obviate the extension step [16]. In particular, the region-based force $F_{r}$ is computed as

$$
F_{r}=\sum_{\mathrm{r}=1}^{2}\left(M_{\mathrm{r}}-\hat{M}_{\mathrm{r}}\right) ; M_{\mathrm{r}}=\frac{\int_{\mathbb{R}^{d}} f_{\mathrm{r}}(x) g\left(a^{-1}(x-y)\right) \mathrm{d} y}{\int_{\mathbb{R}^{d}} C_{\mathrm{r}}(x) g\left(a^{-1}(x-y)\right) \mathrm{d} y+\epsilon},
$$

where $d$ is the dimension of image $f, g$ is a lowpass filter with scale parameter $a, M_{\mathrm{r}}$ is the mean intensity of region $\mathrm{r}$, the region inside (or outside) the current contour, $\hat{M}_{\mathrm{r}}$ is the model mean intensity, $f_{\mathrm{r}}$ is the portion of the image contained in $\mathrm{r}, C_{\mathrm{r}}$ is the binary mask of $\mathrm{r}$ and $\epsilon>0$ is a small correction factor to avoid dividing by zero.

As $F_{r}$ is cast as a convolution, we can use a FFT to expedite the computation. The level-set function at iteration $i$ is evolved as $\phi^{(i+1)}=\phi^{(i)}+\lambda_{r}^{(i)} F_{r}^{(i)}$. As a transformation is used to evolve an active contour with the potential of being applied at multiple scales, we call this MSAC transformation. Applying $F_{r}$ at a fixed scale $a$, we were able to improve the runtime by an order of magnitude, without any degradation in the segmentation quality. The voting-based segmentation using MSAC transformation completed in roughly $1 \mathrm{hr}$.

\section{EXPERIMENTAL RESULTS}

Data Sets. The dataset—MRI of a child brain—was made available to us by the VISTA laboratory at Stanford University [17]. It consists of 400 images -100 in the coronal plane, 140 in the sagittal and 160 in the axial sectioning planes-each of size $217 \times 180$ pixels. The VISTA lab also provided us with human expert-based segmentation for the left hemisphere of the child brain that we then used as "ground truth" to evaluate our algorithm.

Qualitative Analysis. The images shown in Fig. 2 overlay the white matter regions using our technique (red channel) over the manual segmentation (green channel) for selected slices in the coronal, axial and sagittal planes. Yellow pixels indicate regions where the proposed technique and the human expert agree on a white matter labeling, while red and green pixels represent over-segmentation and under-segmentation respectively. One of the major advantages of voting-based STACS over segmentation using only one axis is the voting scheme is able to pick up white-matter regions at the extremities of the MR image while these are often missed in single-axis segmentation. Fig. 3 shows the segmentation results using (a) coronal segmentation and (b) voting-based segmentation for one of the peripheral axial slices. Clearly, coronal segmentation entirely misses the entire white matter region in this slice while voting-based segmentation is able to identify it quite accurately.

Quantitative Analysis. While the qualitative results demonstrate the effectiveness of the proposed technique, we define the following metrics to quantify how well it agrees with the "ground truth":

$M_{1}$ : Percentage of white matter voxels in the "ground truth" labeled correctly as white matter by the voting-based STACS.

$M_{2}$ : Percentage of nonwhite matter voxels in the "ground truth" labeled incorrectly as white matter by the voting-based STACS. 


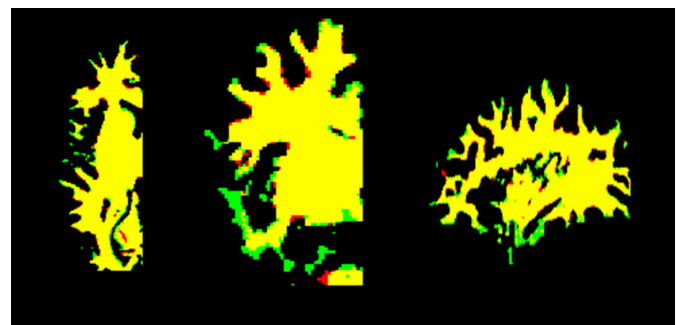

Fig. 2. Qualitative results for selected slices in the axial, coronal and sagittal planes. Yellow indicates the pixels labeled correctly as white matter. Red corresponds to the false positives and green to the false negatives.
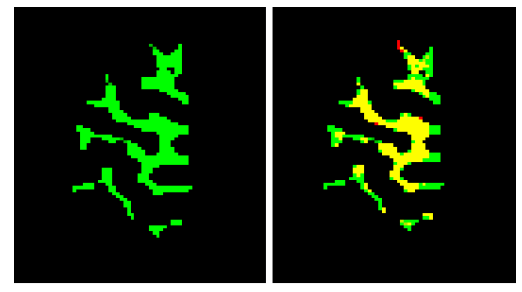

Fig. 3. A peripheral slice in the coronal plane segmented using (a) coronal segmentation only and (b) voting-based segmentation.

$M_{3}$ : Area Similarity is the ratio of the area of the intersection of STACS segmentation and the ground truth and the area of their union. Typically, an $A S \geq 70 \%$ is considered excellent agreement with the ground truth [10].

Table 1 shows the metrics obtained for four possible implementations: voting-based segmentation (Voting), segmentation based on axial slices only (Axial), segmentation based on coronal slices only (Coronal) and segmentation based on sagittal slices only (Sagittal). From the table we note that the voting-based technique achieves the best performance on each metric.

\begin{tabular}{lccc}
\hline & $M_{1}[\%]$ & $M_{2}[\%]$ & $M_{3}[\%]$ \\
\hline Axial & 86.26 & 2.32 & 82.83 \\
Coronal & 89.02 & 2.55 & 80.76 \\
Sagittal & 86.21 & 1.79 & 81.92 \\
Voting & $\mathbf{8 9 . 1 3}$ & $\mathbf{1 . 6 3}$ & $\mathbf{8 5 . 0 7}$ \\
\hline
\end{tabular}

Table 1. Quantitative measures of the segmentation performance of voting-based STACS.

\section{CONCLUSIONS}

We show how a STACS-based segmentation technique with a majority voting procedure can be used to segment white matter in 3D brain MR images. The runtime of the algorithm can be improved using the MSAC transformation. The initial results from an implementation of the proposed technique are encouraging and make a strong case for further research. Opportunities for future work include: incorporating other forces (such as one that uses multiscale edge information) in the MSAC transform framework to further enhance segmentation, devising a better performing voting strategy and plugging the segmentation results back into mrGray to obtain flattened $2 \mathrm{D}$ gray matter representations to help address the physiological questions underlying the problem.

Acknowledgments: We gratefully acknowledge Dr. Michal-Ben Shachar Chechik and Prof. Brian A. Wandell at Stanford University for providing us the data for the analysis and insightful discussions.

\section{REFERENCES}

[1] R. F. Dougherty, V. M. Koch, A. A. Brewer, B. Fischer, J. Modersitzki, and B. A. Wandell, "Visual field representations and locations of visual areas V1/2/3 in human visual cortex," Journ. Vision, vol. 3, pp. 586598, 2003.

[2] B. A. Wandell, "Computational neuroimaging of human visual cortex," Ann. Rev. Neuroscience, vol. 10, no. 22, pp. 145-173, 1999.

[3] N. Gogtay, J. N. Giedd, L. Lusk, K. M. Hayashi, D. Greenstein, A. C. Vaituzis, T. F. Nugent III, D. H. Herman, L. S. Clasen, A. W. Toga, J. L. Rapoport, and P. M. Thompson, "Dynamic mapping of human cortical development during childhood through early adulthood," Proc. Nat. Acad. Sci. of USA, vol. 101, no. 21, pp. 8174-8179, 2004.

[4] H. Zaidi, T. Ruest, F. Schoenahl, and M. L. Montandon, "Comparative assessment of statistical brain MR image segmentation algorithms and their impact on partial volume correction in PET," NeuroImage, vol. 32, pp. 1591-1607, 2006.

[5] N. Kovačević, N. J. Lobaugh, M. J. Bronskill, B. Levine, A. Feinstein, and S. E. Black, "A robust method for extraction and automatic segmentation of brain images," NeuroImage, vol. 17, pp. 1087-1100, 2002.

[6] J. R. J. Alaniz, V. M. Bañuelos, and O. Y. Suárez, "Data-driven brain MRI segmentation supported on edge confidence and a priori tissue information," IEEE Trans. Med. Imag., vol. 25, no. 1, 2006.

[7] J. S. Duncan, X. Papademetris, J. Yang, M. Jackowski, X. Zeng, and L. H. Staib, "Geometric strategies for neuroanatomic analysis from MRI," NeuroImage, vol. 23, pp. S34-S45, 2004.

[8] E. D. Angelini, T. Song, B. D. Mensh, and A. F. Laine, "Brain MRI segmentation with multiphase minimal partitioning: A comparative study," Int. Journ. Biomed. Imaging, vol. 2007, no. 10526, 2007.

[9] C. Pluempitiwiriyawej, J. M. F. Moura, Y.-J. L. Wu, and C. Ho, "STACS: A new active contour scheme for cardiac MR image segmentation," IEEE Trans. Med. Imag., vol. 24, no. 5, pp. 593-603, May 2005.

[10] L. Coulot, H. E. Kirschner, A. Chebira, J. M. F. Moura, J. Kovačević, E. G. Osuna, and R. F. Murphy, "Topology preserving STACS segmentation of protein subcellular location images," in Proc. IEEE Int. Symp. Biomed. Imaging, Arlington, VA, Apr. 2006, pp. 566-569.

[11] P. C. Teo, G. Sapiro, and B. A. Wandell, "Creating connected representations of cortical gray mater for functional MRI visualization," IEEE Trans. Med. Imag., vol. 16, no. 6, pp. 852-863, 1997.

[12] "HOPES: Huntington's Outreach Project for Education, at Stanford," http://www.stanford.edu/group/hopes/basics/braintut/f_ab09whtgrymt.gif.

[13] B. A. Wandell, "Segmentation and Flattening Toolbox Web Site," http://white.stanford.edu/ brian/mri/segmentUnfold.htm.

[14] T. F. Chan and L. A. Vese, "Active contours without edges," IEEE Trans. Image Proc., vol. 10, no. 2, pp. 266-277, Feb. 2001.

[15] F. Gibou and R. Fedkiw, "A fast hybrid k-means level set algorithm," in Proc. Int. Conf. on Stat., Math. and Related Fields, Nov. 2005.

[16] G. Srinivasa, M. C. Fickus, and J. Kovačević, "Multiscale active contour transformations for the segmentation of fluorescence microscope images," in Proc. SPIE Conf. Wavelet Appl. in Signal and Image Proc., San Diego, CA, Aug. 2007, vol. 6701:18, pp. 1-15.

[17] "VISTA: The vision, imaging science and technology activities Lab at Stanford web site," http://white.stanford.edu/. 